Short reports

\title{
Successful treatment of intracaval and atrial extension of Wilms' tumour by chemotherapy
}

\author{
Semha Berberoğlu, Canan Akyüz, Münevver Büyükpamukçu
}

\begin{abstract}
Summary
A patient presenting with advanced Wilms' tumour was diagnosed as having inferior vena cava involvement with tumour thrombus extending up to the right atrium, and was treated with preoperative chemotherapy (vincristine, actinocymin D, epirubicin). Atrial and inferior vena cava thrombus disappeared and he underwent a successful nephrectomy.
\end{abstract}

Keywords: pre-operative chemotherapy, intravascular tumour, thrombus, Wilms' tumour

Progress in the treatment of Wilms' tumour is one of the most significant achievements in the field of paediatric oncology. Current combination multimodal therapy has produced a twoyear disease-free survival rate approaching $86 \%{ }^{1}$ Renal vein involvement (extrarenal) is found in $11.3 \%$ of patients and further spread into the inferior vena cava and/or atrium occurs in an additional $4 \% .^{1,2}$ The surgical management of the Wilms' tumour with vascular extension is associated with considerable morbidity. ${ }^{2,3}$ Pre-operative chemotherapy seems to be gaining favour as the first line therapy in the management of Wilms' tumour. ${ }^{2,4-7}$ There are several reports of chemotherapy being used to manage intravascular tumours, especially in Europe and in some North American centres, following the International Society of Pediatric Oncology protocols. ${ }^{1,2,5,8}$ Those centres following the National Wilms' Tumour Study guidelines still prefer surgery before chemotherapy. ${ }^{9}$ The current report describes our experience with a patient who presented with Wilms' tumour and involvement of the inferior vena cava and right atrium.

\section{Case report}

Ankara Oncology Hospital, Mesa Koru Sitesi, Sedir Sok $2 / C$, 06798 Ankara, Turkey $S$ Berberoğlu

\section{Department of} Paediatric Oncology, Hacettepe University, Institute of Oncology, 06100 Ankara, Turkey C Akyüz

M Büyükpamukçu

Accepted 17 January 1996 enlargement and partial displacement of the right kidney by a large mass. There was distortion and depression of the calyces. The
A four-year-old boy presented with a history of abdominal distention and weight loss of three months duration, and a four-day history of fark coloured urine. On physical right upper and lower quadrants of the abdomen. The remainder of the examination was except for a blood pressure of $130 / 90 \mathrm{mmHg}$ and a mesocardiac systolic ejection. Liver functions of the patient were normal. An intravenous pyelogram showed left kidney was normal. Abdominal ultrasound revealed a homogeneous solid mass arising from the upper portion of the right kidney and crossing the midline and extending to the left kidney. A thrombus was seen in the retrohepatic portion of the inferior vena cava, extending into the right atrium. There was ascites in the abdomen. On thoracic computed tomography (CT), there were multiple metastatic nodules in the right lung. Echocardiographic examination defined a decreased ejection fraction of about $36 \%$ and decreased function of the left ventricle. There was a solid mass in the right atrium (figure).

The patient was considered to have a stage IV Wilms' tumour and underwent a double course of five daily doses of actinomycin D $\left(0.45 \mathrm{mg} / \mathrm{m}^{2}\right)$ and weekly doses of vincristine $\left(1.5 \mathrm{mg} / \mathrm{m}^{2}\right)$ for eight weeks, and epirubicin $\left(50 \mathrm{mg} / \mathrm{m}^{2}\right)$ every three weeks according to the protocol 9 of the International Society of Pediatric Oncology. The following month, thoracoabdominal CT showed that there were no metastatic nodules in the lungs. Repeat echocardiography after three months revealed that there was no solid mass in the right atrium and the ejection fraction was $42 \%$. Repeat ultrasound revealed some decrease in tumour size and thrombus in the inferior vena cava. The patient then underwent right total nephrectomy. At operation the inferior vena cava was found to be infiltrated by tumour mass. Histopathological examination revealed diffuse necrosis and microcalcifications in the tumour mass. There was no tumoural infiltration in the capsule, ureter, and the lymph nodes. Postoperatively the patient received $1050 \mathrm{cGy}$ radiotherapy to both lungs and $1050 \mathrm{cGy}$

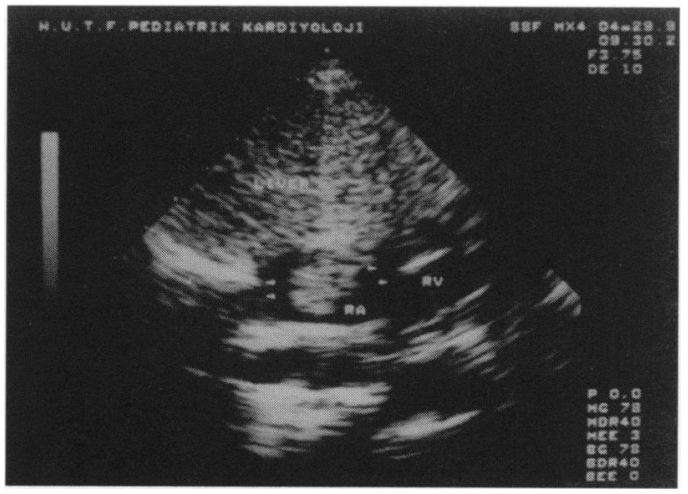

Figure Echocardiogram showing a mass of about $16 \times 9 \mathrm{~mm}$ in the right atrium. RA, right atrium; RV, right venticle 
radiotherapy to the right renal fossa. Three courses of actinomycin $\mathrm{D}\left(0.45 \mathrm{mg} / \mathrm{m}^{2}\right)$ for five days, vincristine $1.5 \mathrm{mg} / \mathrm{m}^{2}$ weekly, and epirubicin $50 \mathrm{mg} / \mathrm{m}^{2}$ every six weeks were given. Echocardiography eight months after presentation showed an ejection fraction of $57 \%$ with no atrial mass. On the abdominal ultrasound there was still a tumour thrombus in the retrohepatic portion of the inferior vena cava. Repeat thoracic CT and abdominal ultrasound 11 months after presentation were normal and there was no thrombus in the inferior vena cava. On ultrasonographic examination two years after presentation, no inferior vena cava thrombus could be detected.

\section{Discussion}

There are several reports in the literature of pre-operative treatment of inferior vena cava or atrial tumour extension in Wilms' tumour patients. ${ }^{4-7,10,11}$ It has been stated that preoperative chemotherapy can successfully shrink the size of the tumour and intravascular tumour extension.

Primary surgical management is appropriate when technically feasible. ${ }^{4-7,10}$ In the US, most patients with intracaval or atrial tumour extension continue to be treated with primary nephrectomy. ${ }^{1,3}$ Pre-operative chemotherapy has been used predominantly for patients with inoperable tumours or bilateral disease. ${ }^{12}$

No particular surgical procedure is associated with an increased death rate but there is an increased rate of surgical complications; such as operative blood loss, wound infection, chylothorax or chylous ascites, intra-operative hypotension, tumour embolism, congestive heart failure and cardiac arrest. ${ }^{1,3,13}$ In our patient, intracardiac extension of the tumour was eradicated with pre-operative chemotherapy, the inferior vena cava thrombus was cleared completely at the end of 18 months chemotherapy, and the lung metastases also disappeared. It is difficult to compare the survival rate of patients given pre-operative therapy with that of patients treated by conventional therapy. We believe that, although surgical management is not associated with an increased death rate, it is a

1 Nakayama DK, Delorimier AA, O'Neil JA. Intracardiac extension of Wilms' tumor. A report of the National Wilms' Tumor Study. Ann Surg 1986; 204: 693-7.

2 Ritchey ML, Kelalis PP, Haase GM. Preoperative therapy for intracaval and atrial extension of Wilms' tumor. Cancer 1993; 71: 4104-10.

3 Ritchey ML, Kelalis PP, Breslow N. Intracaval and atria involvement with nephroblastoma: review of National Wilms Tumor Study-3. 7 Urol 1988; 140: 1113-8.

4 Oberholzer HF, Falkson G, De Jager LC. Successful management of inferior vena cava and right atrial nephroblastoma tumor thrombus with preoperative chemotherapy. Med Pediatr Oncol 1992; 20: 61 - 3 .

5 Bray GL, Pendergrass TW, Schaller RT, Kiviat N, Beckwith JB. Preoperative chemotherapy in the treatment of with JB. Preoperative chemotherapy in the treatment of aspiration biopsy. Am $\mathcal{F}$ Pediatr Hermatol Oncol 1986; 8: aspiration.

6 Bracken RB, Sutow WW, Jaffe N, Ayala A, Guarda L. Preoperative chemotherapy for Wilms' tumor. Urology 1982 19: $55-60$

7 Kogan SJ, Marans H, Santorineau M, Schneider K, Reda E Levitt $B$. Successful treatment of renal vein and vena caval extension of nephroblastoma by preoperative chemotherapy. $\mathcal{F}$ Urol 1986; 136: 312-7.
Table Treatment results of the National Wilms' Tumor Study-3 $3^{14}$

\begin{tabular}{lll}
\hline & \multicolumn{2}{c}{ Disease-free survival } \\
\cline { 2 - 3 } Stage & 2 years (\%) & 4 years (\%) \\
\hline I (favourable histology) & 98 & 98 \\
II (favourable histology) & 96 & 94 \\
III (favourable histology) & 91 & 88 \\
IV (favourable histology) & 88 & 82 \\
Anaplastic I & 89 & 89 \\
Anaplastic II-IV & 56 & 54 \\
\hline
\end{tabular}

\section{Treatment recommendations for} Wilms' tumor ${ }^{14}$

Stage I (favourable histology): surgery, no radiotherapy, actinomycin $D$ +vincristine for 6 months ${ }^{\star}$

Stage I anaplastic (unfavourable histology): surgery, no radiotherapy, actinomycin D+ vincristine for 6 months

Stage II (favourable histology): surgery, no radiotherapy, intensive actinomycin $\mathrm{D}+$ vincristine for 15 months

Stage III (favourable histology): surgery, $1080 \mathrm{cGy}$ to tumour bed, actinomycin D+ vincristine+adriamycin for 15 months

Stage IV (favourable histology): surgery, $1080 \mathrm{cGy}$ to tumour bed, actinomycin D+ vincristine+adriamycin for 15 months

Stage II-IV (unfavourable histology): surgery, radiotherapy, actinomycin $\mathrm{D}$ +vincristine+ adriamycin for 15 months

Babies 11 months of age or younger should receive half the recommended dose of all drugs

* May require only 10 weeks chemotherapy, but results not sufficienctly conclusive for definite recommendation.

formidable process, and can be associated with considerable morbidity. So if technically feasible, primary surgery may be appropriate, but in patients with dense adherence or invasion of the inferior vena cava, or when the tumour extends into the heart we recommend using pre-operative chemotherapy and/or radiation therapy (box). As a result, surgical morbidity will decrease during the subsequent nephrectomy and thrombus removal, or there will be no need for major surgical procedures.

8 Murphy DA, Rabinovitch H, Chevalier L, Virmani S. Wilms' tumor in right atrium. Am $\mathcal{F}$ Dis Child 1973; 126: $210-1$

9 Leape LL, Breslow NE, Bishop HC. The surgical treatment of Wilms' tumor. Results of the National Wilms' Tumor Study. Ann Surg 1978; 187: $351-6$.

10 Wagget J, Koop CE. Wilms' tumor: preoperative radiotherapy and chemotherapy in the management of massive tumors. Cancer 1970; 26: $338-40$.

11 Dykes EH, Marwaha RK, Dicks-Mireaux C, Sams V, Risdon RA, Ransley PG. Risks and benefits of percutaneous biopsy and primary chemotherapy in advanced $W^{\prime}$ ilms' tumour. F Pediatr Surg 1991; 26: 610-2.

12 Montgomery BT, Kelalis PP, Blute ML, Bergstralh EJ, Beckwith JB, Norkool P. Extended follow-up of bilateral Wilms' tumor: results of the National Wilms' Tumor Study. Wilms' tumor: results of the

13 Ritchey MI Kelalis PP, Breslow N, Etzioni R, Evans I, Haase GM. Surgical complications following nephrectomy Haase GM. Surgical complications following nephrectomy for Wilms' tumor: a report of National Wilm

14 Study-3. Surg Gynecol Obstet 1993; 175: 507-14. 14 Lanzkowsky $P$. Wilms' tumor. In: Lanzkowsky $P$, ed. Churchill-Livingstone, 1995; pp 445-7. 